\title{
Subjective and objective accommodation of the Crystalens Advanced Optics (AO) in patients 6 months after bilateral implantation
}

\author{
Marcus Walckling, Ria Beck, Oliver Stachs, Rudolf F. Guthoff \\ Department of Ophthalmology, University of Rostock, Rostock, Germany
}

\begin{abstract}
BACKGROUND: The aim of this study was to analyze and compare objective and subjective accommodation in patients after bilateral implantation of the FDA-approved accommodating Crystalens Advanced Optics (AO).

MATERIAL AND METHODS: This study was performed on 8 eyes of 4 patients. The subjective accommodation was measured by monocular and binocular defocus curves under photopic and mesopic conditions. The objective accommodation was analyzed using partial coherence interferometry (PCI) and wavefront aberration. Pupil diameter at different set-ups and refraction were derived from wavefront data.

RESULTS: Uncorrected and corrected distance and uncorrected near visual acuity improved significantly following implantation of the Crystalens AO in all eyes. Only one patient needed spectacles to correct distance visual acuity. Mean distance uncorrected visual acuity was $0.05 \pm 0.06 \log$ MAR. All patients were able to read newspapers without spectacles. Mean near uncorrected visual acuity was $0.17 \pm 0.12 \log M A R$. Subjective accommodative amplitude under photopic conditions was significantly better binocularly than monocularly $(\mathrm{p}=0.03)$ and was significant worse monocularly under mesopic conditions than photopic conditions $(\mathrm{p}=0.016)$. No significant changes in anterior chamber depth were observed by PCI, with a fixation on optical stimuli at different distances. There were also no significant changes in the wavefront aberrations between the different set-ups. Pupil diameter increased significantly in the near set-up under mesopic conditions.

CONCLUSIONS: No objective change in lens configuration was observed under different set-ups. Due to a variety of factors involved in pseudoaccommodation (e.g. pupil diameter, astigmatism, and multifocality), the subjective accommodative amplitude tends to be overestimated relative to objective measurements. No real accommodative action was observed.
\end{abstract}

KEY WORDS: accommodation; Crystalens; presbyopia correction

Ophthalmol J 2018; Vol. 3, No. 2, 29-36

\section{INTRODUCTION}

Ongoing developments in cataract surgery and intraocular lens (IOL) technology currently enable good visual acuity to be achieved almost as a matter of course following removal of the cloudy lens and implantation of an artificial lens in most patients who are free from ocular co-morbidities. One by no means inconsiderable side-effect of the proce- dure is a loss of accommodation, even though most patients have already lost this ability long before cataract surgery [1]. The clinical research community and industry alike have made it their goal to enable pseudophakic patients to achieve as much spectacle independence as possible. To this end, a range of strategies has been pursued. Most of these currently involve the use of multi-focal lenses 
that incorporate a distance and a near range. However, such lenses have adverse side-effects, such as glare [2] and reduced contrast sensitivity [3]. An altogether different approach is embodied in the Crystalens AO, an FDA-approved "accommodative" IOL (Bausch \& Lomb, Rochester, NY/USA). This model was developed following reports that the ciliary muscle retains its ability to contract even in old age [4]. Previous studies have shown that pharmacologically-induced contraction of the ciliary muscle is associated with the movement of different models of IOLs in pseudophakic eyes [5]. This posterior to anterior translocation has been intentionally exploited in the design of the Crystalens $\mathrm{AO}$. According to the manufacturer, in addition to the movement already referred to, a change in the curvature of the lens (a phenomenon is known as "arching") also plays a role in the functionality of the optic. In addition, there is a group of patients who, despite having a monofocal IOL, achieve good spectacle-independent distance and near visual acuity. Pseudoaccommodation (which is contributed to by factors such as miosis and slight myopic astigmatism that are independent of the implant model used) plays a role in these patients. The aim of this study was to investigate subjective accommodation and objective signs of the accommodative action of the IOL in patients after bilateral implantation of the Crystalens AO.

\section{MATERIALS AND METHODS}

The Crystalens AO was implanted into 8 eyes of 4 patients (aged 64-74 years) at the Department of Ophthalmology, University of Rostock, Germany. It was performed in accordance with the Declaration of Helsinki. Exclusion criteria were ocular co-morbidities and corneal astigmatism greater than 0.75 diopters. After standard phacoemulsification, as recommended by the manufacturer, with a capsulorhexis of $5.5 \mathrm{~mm}$, the IOL was implanted through a clear corneal incision created at the steepest meridian so as to be as astigmatically neutral as possible. The capsule was polished extensively following removal of the cortex. Postoperative treatment was performed with topical ofloxacin (Bausch \& Lomb) and prednisolone (Dr Winzer Pharma, Berlin, Germany). Informed consent was obtained prior to surgery. The refractive power of the IOL was calculated using the SRK/T formula. Emmetropia was targeted. The postoperative follow up was 6 months.

\section{PREOPERATIVE EXAMINATION}

The preoperative examination included tests for distance visual acuity (ETDRS chart at $4 \mathrm{~m}$ ) and near visual acuity (Jäger chart at $40 \mathrm{~cm}$ ), detailed slit-lamp examination of the anterior segment, as well as funduscopy. Topographic corneal measurements were performed with a Pentacam (Oculus, Wetzlar, Germany) to filter out patients with corneal astigmatism greater than 0.75 diopters, and axial lengths were determined using an IOL Master (Zeiss-Meditec, Jena, Germany). The intrcular pressure was measured by applanation tonometry.

\section{PARTIAL COHERENCE INTERFEROMETRY}

Pseudophakic anterior chamber depth (ACD) was measured with the AC-Master (Zeiss-Meditec, Jena, Germany), a modification of the IOL Master, using the principle of partial coherence interferometry (PCI). The non-measured fellow eye was occluded, and the patient fixated on a distance target and a near target, both provided within the device. The mean value from at least 3 measurements was calculated. As well as determining the pseudophakic ACD of the eye under various stimuli, attempts to measure accommodation in pseudophakic eyes have also been made using video-skiascopy [6] and autorefractors [7]. Ultrasound may be employed as an alternative to laser interferometry for measuring $\mathrm{ACD}$, but the resolution and precision of $\mathrm{PCI}$ are higher by a factor of 20 than with conventional ultrasound [8].

\section{WAVEFRONT ABERROMETRY}

Aberrations were measured using the iTrace aberrometer (Tracey Technologies, Houston, Texas), which utilizes the principle of ray tracing. The major advantage of the aberrometer is that the patient can fixate on targets through the same aperture as that through which the measurement is simultaneously conducted. Here, too, monocular measurements were performed and the non-measured eye was occluded. For distance measurements, the patient fixated on an ETDRS chart at $4 \mathrm{~m}$ and for near measurements on a Jäger panel at $40 \mathrm{~cm}$. Distance measurements were performed at an illumination of $30 \mathrm{~cd} / \mathrm{m}^{2}$, and the near measurements at $30 \mathrm{~cd} / \mathrm{m}^{2}$ and $3 \mathrm{~cd} / \mathrm{m}^{2}$. The aberrometer was used in automatic mode to guarantee precise centring of the measured field. In purely manual mode there would have been the potential for the measured field to be decentralized, leading to measurement errors due to the configuration of the Crystalens 
AO (a central elevation of 3 microns and an area of $1.5 \mathrm{~mm}$ ). The $0-44^{\text {th }}$ order wavefront error was extracted and distance and near measurements were statistically compared. In addition to recording the wavefront error, the aberrometer also determined pupil size.

\section{VISUAL ACUITIES}

Uncorrected distance visual acuity (DVA), distance-corrected visual acuity (DCVA), intermediate visual acuity (IVA), distance-corrected intermediate visual acuity (DCIVA), near visual acuity (NVA) and distance-corrected near visual acuity (DCNVA) were determined monocularly for all 8 eyes and binocularly. Distance measurements were performed using the ETDRS visual acuity chart at $4 \mathrm{~m}$, intermediate visual acuity using an intermediate visual acuity chart at $80 \mathrm{~cm}$ (Bausch $\&$ Lomb); near visual acuity under photopic (illumination: $30 \mathrm{~cd} / \mathrm{m}^{2}$ ) and mesopic (illumination: $3 \mathrm{~cd} / \mathrm{m}^{2}$ ) conditions was determined with Jäger charts (Bausch \& Lomb) at $40 \mathrm{~cm}$.

\section{DEFOCUS CURVES}

The best-corrected distance visual acuity (DCVA) was determined. Defocus was conducted in randomized 0.5 diopters (D) steps [9] to $+3 \mathrm{D}$ in the positive direction and to $-5 \mathrm{D}$ in the negative direction. Monocular defocus curves were obtained at an illumination of $30 \mathrm{~cd} / \mathrm{m}^{2}$ and $3 \mathrm{~cd} / \mathrm{m}^{2}$. Binocular defocus was also tested under these conditions. To eliminate any learning effect, two different ETDRS tables were presented and patients were asked to read the lines forward or backwards alternately.

\section{STATISTICAL ANALYSIS}

For statistical reasons, mean values for both eyes of each patient were calculated from the individual measurements, and these mean values were analyzed using Student's t-test for dependent samples.

\section{RESULTS \\ VISUAL OUTCOMES}

At 6 months after implantation all patients showed improved distance visual acuity (mean pre-operative: $0.36 \pm 0.09 \log M A R$; mean post-operative: $-0.08 \pm 0.05 \log$ MAR). This improvement was statistically significant $(\mathrm{p}=0.005)$. The mean spherical equivalent for all eight eyes was $-0.48 \pm 0.39 \mathrm{D}$, and the mean IOL power of all implanted lenses was $19.06 \pm 1.4 \mathrm{D}(17.50-20.75 \mathrm{D})$. One patient required distance spectacles. This patient had a correction of $-0.75 \mathrm{sph} /-0.50 \mathrm{cyl} 100^{\circ}$ in the right eye and $-0.25 \mathrm{sph} /-0.75 \mathrm{cyl} 80^{\circ}$ in the left eye and had binocular distance vision of 20/20 without glasses. With this correction, he achieved 20/12.5, which he found to be more comfortable. All patients reported that they were able to read newspapers without spectacles and that they only used reading glasses in conditions of poor light.

Table 1 contains details of the mean visual acuities, with respective standard deviations, for all four patients.

\begin{tabular}{|c|c|c|}
\hline Visual Acuity & & $\log M A R \pm S D$ \\
\hline Distance Uncorrected Monocular & DUCVAm & $0.05 \pm 0.06$ \\
\hline Distance Corrected Monocular & DCVAm & $-0.08 \pm 0.05$ \\
\hline Distance Uncorrected Binocular & DUCVAb & $-0.04 \pm 0.06$ \\
\hline Distance Corrected Bincoular & DCVAb & $-0.12 \pm 0.06$ \\
\hline Intermediate Uncorrected Monocular & UCIVAm & $-0.02 \pm 0.06$ \\
\hline Intermediate Distance Corrected Monocular & DCIVAm & $0.01 \pm 0.06$ \\
\hline Intermediate Uncorrected Binocular & UCIVAb & $-0.07 \pm 0.05$ \\
\hline Intermediate Distance Corrected Binocular & DCIVAb & $-0.07 \pm 0.05$ \\
\hline Near Uncorrected Monocular & UCNVAm & $0.17 \pm 0.12$ \\
\hline Near Uncorrected Binocular & UCNVAb & $0.15 \pm 0.10$ \\
\hline Near Distance Corrected Monocular & DCNVAm & $0.32 \pm 0.03$ \\
\hline Near Distance Corrected Binocular & DCNVAb & $0.23 \pm 0.05$ \\
\hline Near Uncorrected Mesopic Monocular & UCNVAMm & $0.44 \pm 0.04$ \\
\hline Near Uncorrected Mesopic Binocular & UCNVAMb & $0.42 \pm 0.05$ \\
\hline
\end{tabular}

SD - standard deviation 
Comparison of the various visual acuities showed the following statistical relationships: uncorrected binocular distance visual acuity was significantly better than uncorrected monocular distance visual acuity $(\mathrm{p}<0.001)$. Uncorrected monocular near visual acuity under photopic conditions was significantly better than under mesopic conditions $(p=0.02)$; the same was also true for distance-corrected monocular near visual acuity $(\mathrm{p}=0.012)$. Binocular uncorrected near visual acuity under mesopic conditions were significantly worse than binocular uncorrected ( $\mathrm{p}=0.004)$ and distance-corrected $(\mathrm{p}=0.017)$ near visual acuity under photopic conditions.

\section{DEFOCUS CURVES}

Figure 1 compares the mean values for monocular defocus curves under photopic and mesopic conditions. Visual acuity under photopic conditions was significantly better than under mesopic conditions

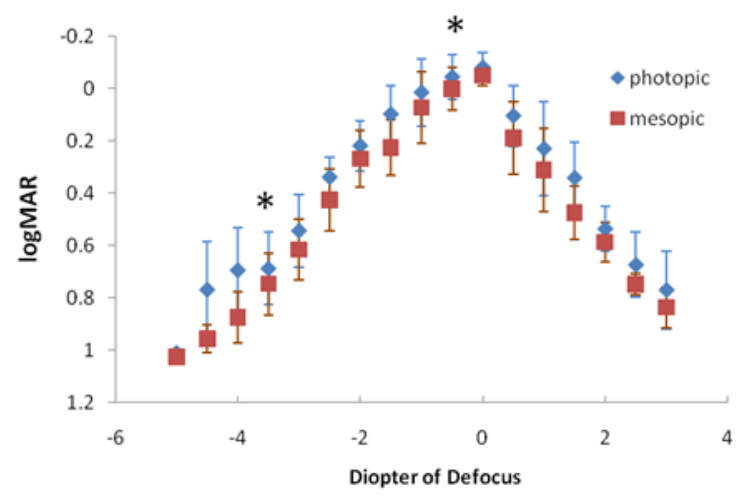

FIGURE 1. Monocular visual acuity at different defocus levels: a comparison under photopic and mesopic conditions $\left({ }^{*}\right.$ denotes statistically significant difference)

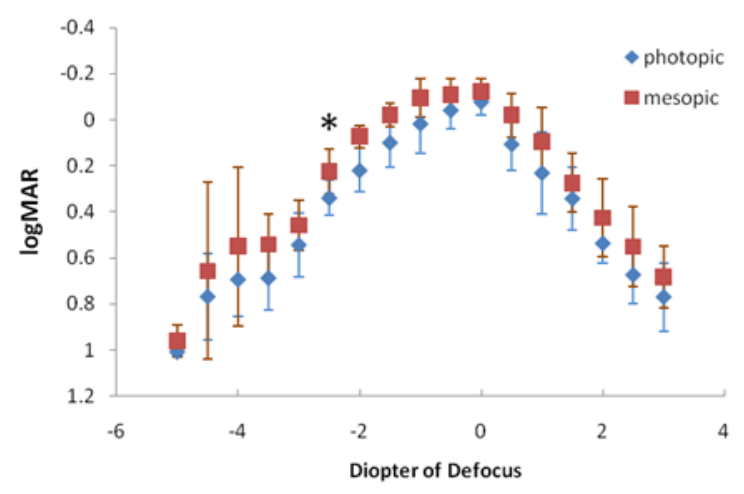

FIGURE 2. Monocular and binocular visual acuity at different defocuses levels under photopic conditions ( ${ }^{*}$ denotes statistically significant difference) at a defocus of $-3.5 \mathrm{D}$ (photopic: $0.68 \pm 0.14 \mathrm{log}$ MAR; mesopic: $0.75 \pm 0.11 \log$ MAR; $P=0.031$ ) and $-0.5 \mathrm{D}$ (photopic: $-0.04 \pm 0.08 \log \mathrm{MAR}$; mesopic: $0.001 \pm 0.08 ; \mathrm{p}=0.048$ ).

Under photopic conditions at a defocus of $-2.5 \mathrm{D}$ (Fig. 2), binocular visual acuity $(0.22 \pm 0.09 \mathrm{log}$ MAR) was significantly better $(\mathrm{p}=0.037)$ than monocular visual acuity $(0.34 \pm 0.08 \log M A R)$.

Figure 3 compares the mean values for monocular and binocular defocus curves under mesopic conditions. Binocular visual acuity was significantly better than monocular visual acuity at a defocus of $-1.5 \mathrm{D}$ (monocular: $0.23 \pm 0.10 \log \mathrm{MAR}$; binocular: $-0.007 \pm 0.07 \log$ MAR, $p=0.039)$ and $-2.5 \mathrm{D}$ (monocular: $0.42 \pm 0.11 \log \mathrm{MAR}$; binocular: $0.30 \pm 0.08 \log$ MAR; $p=0.032$ ).

At a defocus of $+1 \mathrm{D}$ (Fig. 4), the binocular defocus curves were significantly better $(\mathrm{p}=0.005)$ under photopic conditions $(0.09 \pm 0.15 \log \mathrm{MAR})$ than under mesopic conditions $(0.18 \pm 0.16 \log M A R)$.

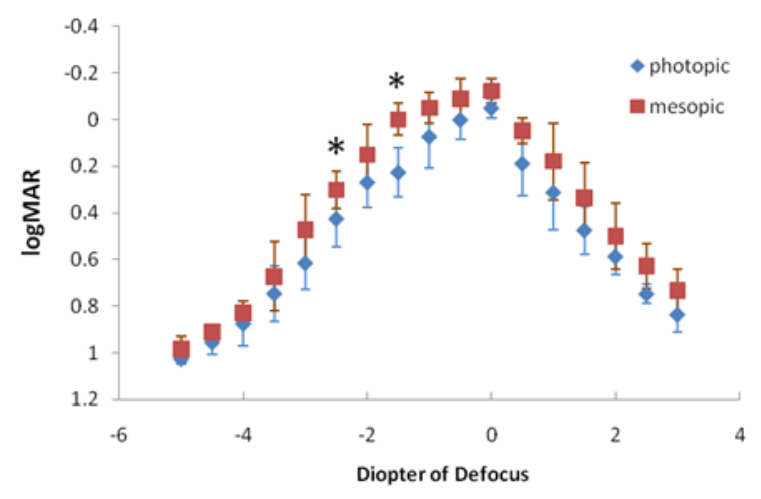

FIGURE 3. Monocular and binocular visual acuity at different defocus levels under mesopic conditions $\left({ }^{*}\right.$ denotes statistically significant difference)

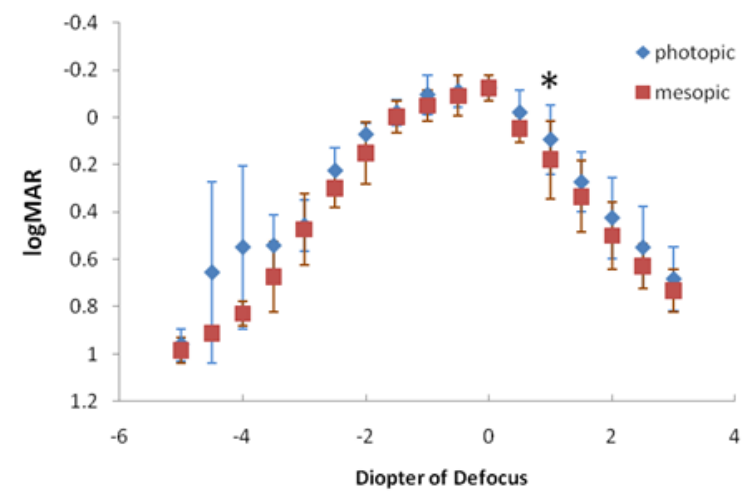

FIGURE 4. Binocular visual acuity at different defocus levels: comparison under photopic and mesopic conditions ( ${ }^{*}$ denotes statistically significant difference) 


\section{OBJECTIVE CHANGE IN REFRACTION (ABERROMETER DATA)}

Refraction in different zones was calculated by aberrometry, and the values recorded in the central zone ( $2 \mathrm{~mm}$ in all patients) were compared for distance and near set-ups. No statistically significant change in the sphere $(\mathrm{p}=0.199)$ or the cylinder $(\mathrm{p}=0.663)$ was observed, with the result that no change in refraction was measured objectively.

\section{SUBJECTIVE AMPLITUDE OF ACCOMIMODATION}

The subjective amplitude of accommodation (Fig. 5) was determined using the defocus curves. The range of defocusing over which the patient's visual acuity remained stable over 2 lines (or $\log$ MAR 0.2) was noted as the amplitude of accommodation. Under photopic conditions, this was significantly worse $(\mathrm{p}=0.03)$ monocularly $(1.75 \pm 0.2 \mathrm{D})$ than binocularly $(2.4 \pm 0.3 \mathrm{D})$, but significantly better $(\mathrm{p}=0.016)$ than under mesopic conditions $(1.25 \pm 0.3 \mathrm{D})$. The binocular amplitude under mesopic conditions was $2 \pm 0.4 \mathrm{D}$, and there was no significant difference by comparison with the monocular mesopic amplitude.

\section{STIMULUS-DRIVEN CHANGE IN ACD IN THE PSEUDOPHAKIC EYE}

The mean ACD was $4.48 \pm 0.14 \mathrm{~mm}$ during fixation on a distance target and $4.49 \pm 0.13 \mathrm{~mm}$ during fixation on a near target. The mean change of $-0.010 \pm 0.008 \mathrm{~mm}$ was not statistically significant $(\mathrm{p}=0.116)$.

\section{CHANGE IN PUPIL DIAMETER}

Pupil size was derived from the aberrometry data: in each case, the horizontal and vertical diameters were measured and the mean was formed. Mean pupil diameter during distance gaze was $3.34 \pm 0.3 \mathrm{~mm}$ under photopic conditions; during fixation on the Jäger chart, it was $3.27 \pm 0.4 \mathrm{~mm}$ under photopic conditions and $3.99 \pm 0.4 \mathrm{~mm}$ under mesopic conditions. The difference between the distance and near set-ups in the photopic range was not significant $(\mathrm{p}=0.52)$. A statistically significant increase in pupil diameter was observed in the near set-up under mesopic conditions $(\mathrm{p}=0.009)$.

\section{WAVEFRONT ABERRATIONS}

Wavefront measurements were performed in all eight eyes during fixation on a distance target (ETDRS chart at $4 \mathrm{~m}$ ) and a near target (Jäger chart at

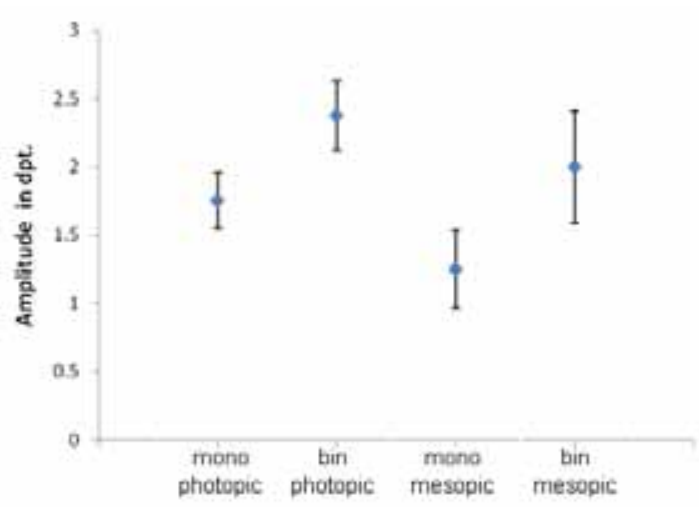

FIGURE 5. Monocular and binocular accommodative amplitude (D) under photopic and mesopic conditions

$40 \mathrm{~cm}$ ). The aberrations of both eyes for each patient were averaged and the mean values for all patients were compared under different conditions. Comparison of mean aberrations during distance vs. near fixation revealed no statistically significant differences, although trends were noted for Z 14 ( $p=0.066)$, Z 17 ( $p=0.084)$, and Z 18 ( $p=0.066)$, respectively. Similarly, no statistically significant differences were found when mean aberrations were compared under near mesopic $v s$. near photopic conditions.

\section{DISCUSSION}

The newest strategies for spectacle independence in pseudophakic patients include multifocal lenses, accommodating lenses and monovision, all of which have advantages and disadvantages. Halo and glare have occurred significantly more often following implantation of multifocal lenses compared with monofocal lenses [10], and in rare cases, these phenomena may even necessitate explantation. Contrast sensitivity is also impaired to some degree. A further option is offered by the concept of monovision, which demands high-precision biometry because stereoscopic vision is diminished beyond a certain amount of anisometropia. According to Hayashi et al. [11], approximately $1.50 \mathrm{D}$ of anisometropia is regarded as optimal for successful monovision without degradation of stereoscopic vision. Another concept is mini-monovision following implantation of the Tecnis 1-Piece IOL (Abbott Medical Optics Inc., Santa Ana, CA), Wittpenn [12] has reported on 26 patients, $89 \%$ of whom had uncorrected binocular distance visual acuity of 20/25 or better and $92.3 \%$ had uncorrected near visual acuity of $\mathrm{J} 3$ or better. Target refraction was plano to $-0.25 \mathrm{D}$ 
for the dominant eye, and -0.50 to $-1.00 \mathrm{D}$ for the non-dominant eye.

In the present study of subjective and objective signs of accommodation with the Crystalens AO, distance and near visual acuities were found to be improved in all patients compared to pre-operative status. When the change in ACD was measured during visual stimulation, there was no significant change suggestive of forward movement and, on average, there was minimal, non-significant backward movement. Koeppl et al. [13] also reported a significant backward shift with the predecessor of the Crystalens AO, the AT- 45 , when the ciliary muscle was pharmacologically stimulated with $2 \%$ pilocarpine. However, that effect was pharmacologically induced and therefore considerably greater than the expected effect of visual stimulation in our study. With other implant models, pharmacological stimulation has also been found to be more powerful than visual stimulation. In studies with the 1CU (marketed by HumanOptics as an accommodating IOL), forward movement of $370 \pm 290$ microns was reported after application of $2 \%$ pilocarpine, whereas visual stimulation failed to trigger any significant shift [14].

An increase in the vitreous pressure gradient has also been suggested as a possible cause of this movement. This gradient is also thought to increase in response to contraction of the rectus medialis during the convergence reaction [15]. In the present study, occlusion of the fellow eye for the wavefront and ACD measurements has possibly eliminated this component.

As in other studies with accommodating IOLs [16], subjective accommodation was also determined in the present study. During monocular testing, a significant decrease in the amplitude of accommodation was found under mesopic conditions compared with photopic conditions. This was associated with a significant increase in pupil diameter and a resultant decrease in the depth of field. Binocularity is able to compensate for this effect in part. The subjective range or amplitude of accommodation is usually overestimated relatively to objective measurements with the AC-Master and aberrometer [17]. Objective refraction measurements derived from our aberrometry data did not reveal any statistically significant change in the four patients investigated here.

In addition to pupil size, there is evidence to indicate that other factors also play a role in pseudoaccommodation. Thus, coma-like aberration has been reported to show a significant positive correlation with accommodation or pseudoaccommodation in pseudophakic eyes [18]. The presence of against-the-rule astigmatism has also been shown to have some benefit for near visual acuity [19].

It may be that the Crystalens $\mathrm{AO}$ also generates slight multifocality: centrally the IOL optic includes a 1.5 -mm-diameter modification that adds $3 \mu \mathrm{m}$ of thickness and provides a small degree of negative spherical aberration (information supplied by Bausch \& Lomb, Rochester, NY) [20]. This modification could not be visualized by wavefront aberrometry in the present study. All the aforementioned factors may contribute to good uncorrected near visual acuity, although the objective measurements performed here did not yield evidence to suggest an active accommodative process. In all four patients studied, the reading ability was achieved, with one patient requiring spectacles for distance vision. In the study conducted by Alió et al [21]. comparable optical quality with significantly improved uncorrected near visual acuity was obtained with the Crystalens AO compared with a monofocal lens.

To date, no significant, reproducible axial movement has been detected with the Crystalens AO during visual stimulation, despite good results in terms of near visual acuity. The objective measurements carried out here showed no evidence of an active accommodative action of the Crystalens AO. Strategies such as mini-monovision may provide similar results with monofocal IOLs.

\section{ACKNOWLEDGEMENTS}

The authors wish to thank T. Terwee for the fruitful discussions, and Mr. David Beattie for editorial support in the preparation of this manuscript.

Financial disclaimer: No author has a financial or proprietary interest in any material or method mentioned.

\section{REFERENCES}

1. Duane A. Normal values of the accommodation at all ages. JAMA. 1912; 59(12): 1010-1013, doi: 10.1001/jama.1912.04270090254042.

2. Winther-Nielsen A, Gyldenkerne G, Corydon L. Contrast sensitivity, glare, and visual function: diffractive multifocal versus bilateral monofocal intraocular lenses. J Cataract Refract Surg. 1995; 21(2): 202-207, indexed in Pubmed: 7791063.

3. Haaskjold E, Allen ED, Burton RL. Contrast sensitivity after implantation of diffractive bifocal and monofocal intraocular lenses. J Cataract Refract Surg. 1998; 24(5): 653-658, indexed in Pubmed: 9610448.

4. Bacskulin A, Martin H, Kundt G, et al. [Analysis of the dynamics of the ciliary muscle during accommodation]. Ophthalmologe. 2000; 97(12): 855-859, indexed in Pubmed: 11227158. 
5. Findl O, Kiss B, Petternel V, et al. Intraocular lens movement caused by ciliary muscle contraction. J Cataract Refract Surg. 2003; 29(4): 669-676, doi: 10.1016/S0886-3350(02)01652-8, indexed in Pubmed: 12686233.

6. Langenbucher A, Seitz B, Huber S, et al. Theoretical and measured pseudophakic accommodation after implantation of a new accommodative posterior chamber intraocular lens. Arch Ophthalmol. 2003; 121(12): 1722-1727, doi: 10.1001/archopht.121.12.1722, indexed in Pubmed: 14662592.

7. Cleary G, Spalton DJ, Marshall J. Pilot study of new focus-shift accommodating intraocular lens. J Cataract Refract Surg. 2010; 36(5): 762-770, doi: 10.1016/j.jcrs.2009.11.025, indexed in Pubmed: 20457367.

8. Findl 0 , Drexler W, Menapace R, et al. High precision biometry of pseudophakic eyes using partial coherence interferometry. J Cataract Refract Surg. 1998; 24(8): 1087-1093, indexed in Pubmed: 9719968.

9. Gupta N, Naroo SA, Wolffsohn JS. Is randomisation necessary for measuring defocus curves in pre-presbyopes? Cont Lens Anterior Eye. 2007; 30(2): 119-124, doi: 10.1016/j.clae.2007.02.005, indexed in Pubmed: 17448927.

10. Häring G, Dick H, Krummenauer F, et al. Subjective photic phenomena with refractive multifocal and monofocal intraocular lenses. J Cataract Refract Surg. 2001; 27(2): 245-249, doi: 10.1016/s0886-3350(00)00540-x, indexed in Pubmed: 11226790.

11. Hayashi K, Yoshida M, Manabe SI, et al. Optimal amount of anisometropia for pseudophakic monovision. J Refract Surg. 2011; 27(5): 332-338, doi: 10.3928/1081597X-20100817-01, indexed in Pubmed: 20839664.

12. Wittpenn JJ. Mini-monovision with the Tecnis 1-Piece provides betterthan-expected near vision with minimal compromise at distance. Cataract Refract Surg Today. 2010: 70.
13. Koeppl C, Findl 0, Menapace R, et al. Pilocarpine-induced shift of an accommodating intraocular lens: AT-45 Crystalens. J Cataract Refract Surg. 2005; 31(7): 1290-1297, doi: 10.1016/j.jcrs.2005.03.055, indexed in Pubmed: 16105597.

14. Kriechbaum K, Findl O, Koeppl C, et al. Stimulus-driven versus pilocarpine-induced biometric changes in pseudophakic eyes. Ophthalmology. 2005; 112(3): 453-459, doi: 10.1016/j.ophtha.2004.09.021, indexed in Pubmed: 15745773.

15. Coleman DJ. Unified model for accommodative mechanism. Am J Ophthalmol. 1970; 69(6): 1063-1079, indexed in Pubmed: 5423772.

16. Ossma IL, Galvis A, Vargas LG, et al. Synchrony dual-optic accommodating intraocular lens. Part 2: pilot clinical evaluation. $\mathrm{J}$ Cataract Refract Surg. 2007; 33(1): 47-52, doi: 10.1016/j.jcrs.2006.08.049, indexed in Pubmed: 17189792.

17. Win-Hall DM, Glasser A. Objective accommodation measurements in pseudophakic subjects using an autorefractor and an aberrometer. J Cataract Refract Surg. 2009; 35(2): 282-290, doi: 10.1016/j. jcrs.2008.10.033, indexed in Pubmed: 19185244.

18. Oshika T, Mimura T, Tanaka S. Apparent accommodation and corneal wavefront aberration in pseudophakic eyes. Invest Ophthalmol Vis Sci. 2002; 43(9): 2882-2886, indexed in Pubmed: 12202506.

19. Trindade F, Oliveira A, Frasson M. Benefit of against-the-rule astigmatism to uncorrected near acuity. J Cataract Refract Surg. 1997; 23(1): 82-85, indexed in Pubmed: 9100112.

20. Form and Function: The Crystalens HD. DataLink statistics and clinical experience from the San Francisco users' meeting. Cataract Refract Surg Today. 2009; Suppl: 1-16.

21. Alió JL, Piñero DP, Plaza-Puche AB. Visual outcomes and optical performance with a monofocal intraocular lens and a new-generation single-optic accommodating intraocular lens. J Cataract Refract Surg. 2010; 36(10): 1656-1664, doi: 10.1016/j.jcrs.2010.04.040, indexed in Pubmed: 20870110. 
